Antonio Boccuto and Anna Rita Sambucini, Department of Mathematics, via Vanvitelli 1, 06123 Perugia, Italy, e-mail: tipo@ipguniv.unipg.it, matears@@ipguniv.unipg.it

\title{
ON THE DE GIORGI-LETTA INTEGRAL WITH RESPECT TO MEANS WITH VALUES IN RIESZ SPACES
}

\begin{abstract}
A monotone integral is given for scalar function, with respect to Riesz space values means, and also a necessary and sufficient condition to obtain a Radon-Nikodym density for two means.
\end{abstract}

\section{Introduction}

Integrals like Kurzweil-Stieltjes, Riemann sums and Bochner have been studied in vector lattices by Duchoň, Riečan and Vrábelová, ([11], [21], [22]), Wright ([26], [27]), McGill ([19]), Šipoš ([24]), Maličký ([18]), Cristescu ([8]), Haluška ([15]), Boccuto ([3], [4]), and others.

In this paper we extend to such spaces the monotone integral, given by Choquet in 1953 ([6]), and developed by De Giorgi-Letta ([9]), Greco ([13]), Brooks-Martellotti ([5]), and others ([10], [12], [16], etc.).

Given a mean $\mu: \mathcal{A} \rightarrow R$ and a measurable function $f: X \rightarrow \widetilde{\mathbb{R}}_{0}^{+}$, we say that $f$ is integrable (in the monotone sense) if the following limit exists in $R$.

$$
(o)-\lim _{a \rightarrow+\infty} \int_{0}^{a} \mu(\{x \in X: f(x)>t\}) d t .
$$

For this integral we obtain some elementary properties and we give some Vitalitype theorems. We note that in general this integral is different from the one

Key Words: monotone integral, convergence theorems, Radon-Nikodym theorem

Mathematical Reviews subject classification: Primary: 28A70

Received by the editors November 3, 1995

* Lavoro svolto nell'ambito dello G.N.A.F.A. del C.N.R. 
introduced in [5] for Banach spaces. Finally we prove a version of RadonNikodym-type theorems for the introduced integral (see also [14]).

Our thanks to Prof. D. Candeloro and A. Martellotti for their helpful suggestions.

\section{Preliminaries}

We begin with some definitions.

Definition 2.1. A Riesz space $R$ is called Archimedean if the following property holds.

(2.1.1) For every choice of $a, b \in R$, if $n a \leq b$ for all $n \in \mathbb{N}$, then $a \leq 0$.

Definition 2.2. A Riesz space $R$ is said to be Dedekind complete (resp. $\sigma$ Dedekind complete) if every nonempty (countable) subset of $R$, bounded from above, has supremum in $R$.

The following results are well-known (see [1], [2]).

Proposition 2.3. Every $\sigma$-Dedekind complete Riesz space is Archimedean.

Theorem 2.4. Given an Archimedean (Dedekind complete) Riesz space R, there exists a compact Stonian topological space $\Omega$, unique up to homeomorphisms such that $R$ can be embedded as a (solid) subspace of

$$
\mathcal{C}_{\infty}(\Omega)=\left\{f \in \widetilde{\mathbb{R}}^{\Omega}: f \text { is continuous, and }\{\omega:|f(\omega)|=+\infty\}\right.
$$

is nowhere dense in $\Omega\}$. Moreover if $\left(a_{\lambda}\right)_{\lambda \in \Lambda}$ is any family such that $a_{\lambda} \in$ $R \forall \lambda$ and $a=\sup _{\lambda} a_{\lambda} \in R$ (where the supremum is taken with respect to $R$ ), then $a=\sup _{\lambda} a_{\lambda}$ with respect to $\mathcal{C}_{\infty}(\Omega)$ and the set $\left\{\omega \in \Omega:\left(\sup _{\lambda} a_{\lambda}\right)(\omega) \neq\right.$ $\left.\sup _{\lambda} a_{\lambda}(\omega)\right\}$ is meager in $\Omega$.

Definition 2.5. A sequence $\left(r_{n}\right)_{n}$ is said to be order-convergent (or (o)-convergent) to $r$, if there exists a sequence $\left(p_{n}\right)_{n} \in R$ such that $p_{n} \downarrow 0$ and $\left|r_{n}-r\right| \leq p_{n}, \forall n \in \mathbb{N}$, and we will write $(o)-\lim _{n} r_{n}=r$.

As $\left|r_{n}\right| \leq|r|+p_{1} \forall n$, every (o)-convergent sequence is bounded. We note that, if $R$ is a $\sigma$-Dedekind complete Riesz space, $(o$ )-convergence can be formulated in the following equivalent ways (see also [25]).

Proposition 2.6. A sequence $\left(r_{n}\right)_{n}$, bounded in $R,(o)$-converges to $r$ if and only if $r=(o)-\limsup \sup _{n} r_{n}=(o)-\liminf _{n} r_{n}$, where $(o)-\lim \sup _{n} r_{n}=$ $\inf _{n}\left[\sup _{m \geq n} r_{m}\right], \quad(o)-\liminf _{n} r_{n}=\sup _{n}\left[\inf _{m \geq n} r_{m}\right]$. 
Proposition 2.7. Let $R$ be as above, $\Omega$ as in Theorem 2.4. A bounded sequence $\left(r_{n}\right)_{n}, r_{n} \in R,(o)$-converges to $r$ if and only if the set $\{\omega \in \Omega$ : $\left.r_{n}(\omega) \nrightarrow \rightarrow r(\omega)\right\}$ is meager in $\Omega$.

We recall some fundamental properties of the order convergence (see [25]).

Proposition 2.8. If $\left(r_{n}\right)_{n}(o)$-converges to both $r$ and $s$, then $r \equiv s$. If $\left(r_{n}\right)_{n}(o)$-converges to $r,\left(s_{n}\right)_{n}(o)$-converges to $s$ and $\alpha \in \mathbb{R}$, then $\left(r_{n}+s_{n}\right)_{n}$, $\left(r_{n} \vee s_{n}\right)_{n},\left(r_{n} \wedge s_{n}\right)_{n},\left(\alpha r_{n}\right)_{n},\left(\left|r_{n}\right|\right)_{n}(o)$-converge respectively to $r+s, r \vee s$, $r \wedge s, \alpha r,|r|$.

Definition 2.9. A sequence $\left(r_{n}\right)_{n}$ is said to be (o)-Cauchy if there exists a sequence $\left(p_{n}\right)_{n} \in R$ such that $p_{n} \downarrow 0$ and $\left|r_{n}-r_{m}\right| \leq p_{n}, \forall n \in \mathbb{N}$, and $\forall m \geq n$.

Definition 2.10. A Riesz space $R$ is called (o)-complete if every (o)-Cauchy sequence is (o)-convergent.

The following result holds (see [17], [28]).

Proposition 2.11. Every $\sigma$-Dedekind complete Riesz space is (o)-complete.

We note that there are some cases, in which $(o)$-convergence is not "generated" by a topology. For example, $L^{0}(X, \mathcal{B}, \mu)$, where $\mu$ is a $\sigma$-additive non-atomic positive $\widetilde{\mathbb{R}}$-valued measure. We recall that, in such spaces, $(o)$ convergence coincides with almost everywhere convergence. (Also see [25].)

\section{The Monotone Integral}

Definition 3.1. Let $X$ be any set, $R$ a Dedekind complete Riesz space, $\mathcal{A} \subset$ $\mathcal{P}(X)$ an algebra. $A$ map $\mu: \mathcal{A} \rightarrow R$ is said to be mean if $\mu(A) \geq 0, \forall A \in \mathcal{A}$ and $\mu(A \cup B)=\mu(A)+\mu(B)$ whenever $A \cap B=\emptyset$. A mean $\mu$ is countably additive (or $\sigma$-additive) if $\mu\left(\cap_{n} A_{n}\right)=\inf _{n} \mu\left(A_{n}\right)$, whenever $\left(A_{n}\right)_{n}$ is a decreasing sequence in $\mathcal{A}$, such that $\cap_{n} A_{n} \in \mathcal{A}$.

Given a mapping $f: X \rightarrow \tilde{\mathbb{R}}_{0}^{+}$and a mean $\mu$ as above for all $A \in \mathcal{A}$ and $t \in \mathbb{R}_{0}^{+}$, set $E_{t, A}^{f}$ (or simply $E_{t, A}$, when no confusion can arise) $\equiv\{x \in$ $A: f(x)>t\} ; E_{t}^{f}\left(E_{t}\right) \equiv\{x \in X: f(x)>t\}$; and, for every $t>0$, let $u_{A, f}(t) \equiv \mu\left(E_{t, A}^{f}\right) ; u_{f}(t)=u(t) \equiv \mu\left(E_{t}\right)$.

Definition 3.2. With the same notation as above, we say that a function $f: X \rightarrow \tilde{\mathbb{R}}_{0}^{+}$is measurable if $E_{t}^{f} \in \mathcal{A}, \forall t \in \mathbb{R}^{+}$.

Now we define a Riemann (Lebesgue)-type integral, for maps, defined in an interval of the real line and taking values in a Dedekind complete Riesz space. (For similar integrals existing in the literature, also see [21] and [20].) 
Definition 3.3. Let $a, b \in \mathbb{R}, a<b$, and $R$ be as above. We say that a map $g:[a, b] \rightarrow R$ is a step function if there exist $n+1$ points $x_{0} \equiv a<x_{1}<$ $\ldots<x_{n} \equiv b$ such that $g$ is constant in each interval of the type $] x_{i-1}, x_{i}[(i=$ $1, \ldots, n)$. We say that $g$ is simple if there exist $n$ elements of $R, a_{1}, \ldots, a_{n}$, and $n$ pairwise disjoint measurable sets $E_{i}$ such that $g=\sum_{i=1}^{n} a_{i} \chi_{E_{i}}$. If $g$ is a step (simple) function, we put $\int_{a}^{b} g(t) d t \equiv \sum_{i=1}^{n}\left(x_{i}-x_{i-1}\right) \cdot g\left(\xi_{i}\right)\left[\sum_{i=1}^{n}\left|E_{i}\right| \cdot g\left(\xi_{i}\right)\right]$, where $\xi_{i}$ is an arbitrary point of $] x_{i-1}, x_{i}\left[\left[E_{i}\right]\right.$.

Definition 3.4. Let $u:[a, b] \rightarrow R$ be a bounded function. We call the upper integral (resp. lower integral) of $u$ the element of $R$ given by

$$
\inf _{v \in V_{u}} \int_{a}^{b} v(t) d t\left[\sup _{s \in S_{u}} \int_{a}^{b} s(t) d t\right],
$$

where

$$
\begin{aligned}
& V_{u} \equiv\{v: v \text { is a step (simple) function, } v(t) \geq u(t), \forall t \in[a, b]\} \\
& S_{u} \equiv\{s: s \text { is a step (simple) function, } s(t) \leq u(t), \forall t \in[a, b]\} .
\end{aligned}
$$

We say that $u$ is Riemann (Lebesgue) integrable (or $(R)$, resp. $(L)$-integrable) if its lower integral coincides with its upper integral and, in this case, we call integral of $u$ (and write $\int_{a}^{b} u(t) d t$ ) their common value.

It is easy to check that this integral is well-defined, and is a linear monotone functional, with values in $R$.

The following result holds.

Proposition 3.5. Every bounded monotone map $u:[a, b] \rightarrow R$ is Riemann integrable.

Proof. The proof is almost identical to the classical one.

Now we define an integral for extended real-valued functions with respect to $R$-valued means.

Definition 3.6. Let $X, R, \mu, f: X \rightarrow \widetilde{\mathbb{R}}_{0}^{+}, u=u_{f}$ be as above. We say that $f$ is integrable if the quantity

$$
\int_{0}^{+\infty} u(t) d t \equiv \sup _{a>0} \int_{0}^{a} u(t) d t=(o)-\lim _{a \rightarrow+\infty} \int_{0}^{a} u(t) d t
$$

exists in $R$ where the integral in (3.6.1) is intended as in Definition 3.4. If $f$ is integrable, we denote the element in (3.6.1) by $\int_{X} f d \mu$. A measurable function $f: X \rightarrow \mathbb{R}$ is integrable if both $f^{+}, f^{-}$are integrable and, in this case we set $\int_{X} f d \mu=\int_{X} f^{+} d \mu-\int_{X} f^{-} d \mu$. 
Remark 3.7. We can extend Definition 3.6 when $\mu: \mathcal{A} \rightarrow R$ is any finitely additive bounded map. A measurable function $f$ is integrable if and only if $f$ is integrable with respect to $\mu^{+}, \mu^{-}$, where for every $A \in \mathcal{A}$

$$
\begin{aligned}
& \mu^{+}(A) \equiv \vee_{B \subset A, B \in \mathcal{A}} \mu(B), \\
& \mu^{-}(A) \equiv-\wedge_{B \subset A, B \in \mathcal{A}} \mu(B),
\end{aligned}
$$

and $\mu=\mu^{+}-\mu^{-}$. In this case, we set $\int_{X} f d \mu \equiv \int_{X} f d \mu^{+}-\int_{X} f d \mu^{-}$. (Also see [7].)

An immediate consequence of Definition 3.6 and monotonicity of $\mu$ is the following assertion.

Proposition 3.8. If $f$ is integrable, then for each $A \in \mathcal{A}, \sup _{a>0} \int_{0}^{a} u_{A, f}(t) d t$ exists in $R$ and is denoted by $\int_{A} f d \mu$.

Proposition 3.9. With the same notation as above, if $f$ is integrable, then

$$
\int_{A} f d \mu=\int_{X} f \cdot \chi_{A} d \mu, \quad \forall A \in \mathcal{A} .
$$

Proof. For each fixed $t>0$ and $x \in X$, we have $\left[f \cdot \chi_{A}(x)>t\right]$ if and only if $[x \in A]$ and $[f(x)>t]$. So, $u_{X, f \cdot \chi_{A}} \equiv u_{A, f}$. Thus, the assertion follows.

It is easy to check that this integral is a linear $R$-valued functional and that, for every positive integrable map $f, \int f d \mu$ is a mean.

We now list a number of technical results.

Proposition 3.10. If $f$ is integrable, then $(o)-\lim _{t \rightarrow+\infty} \mu\left(E_{t}\right)=0$ and hence $\mu\left(E_{\infty}\right)=0$, where $E_{\infty} \equiv\{x \in X: f(x)=+\infty\}$.

Proof. For every $t>0$, we have

$$
0 \leq \mu\left(E_{\infty}\right) \leq \mu\left(E_{t}\right)=\frac{\int_{E_{t}} t d \mu}{t} \leq \frac{\int_{E_{t}} f d \mu}{t} \leq \frac{\int_{X} f d \mu}{t} .
$$

Taking the infimum, we obtain $0 \leq \mu\left(E_{t}\right) \leq \inf _{t>0} \frac{\int_{X} f d \mu}{t}=0$.

Proposition 3.11. Let $f: X \rightarrow \tilde{\mathbb{R}}_{0}^{+}$be measurable. Then, $f$ is integrable if and only if $\sup _{n} \int_{X}(f \wedge n) d \mu \in R$, and in this case $\sup _{n} \int_{X}(f \wedge n) d \mu=\int_{X} f d \mu$.

Proof. Fix $n \in \mathbb{N}$ and pick $t<n$. Then $f(x) \wedge n>t$ if and only if $f(x)>t$ and so $\int_{0}^{n} u_{f}(t) d t=\int_{0}^{n} u_{f \wedge n}(t) d t=\int_{0}^{+\infty} u_{f \wedge n}(t) d t=\int_{X}(f \wedge n) d \mu$. So the first part of the assertion follows immediately. Moreover taking the suprema, we get $\sup _{n} \int_{X}(f \wedge n) d \mu=(o)-\lim _{n \rightarrow+\infty} \int_{0}^{n} u_{f}(t) d t=\int_{X} f d \mu$. 
Proposition 3.12. Let $f: X \rightarrow \mathbb{R}_{0}^{+}$be measurable and bounded and set $S_{f}$ (resp. $\left.V_{f}\right) \equiv\{g: X \rightarrow \mathbb{R}: g \leq f, g$ is simple $\}$, (resp. $\{h: X \rightarrow \mathbb{R}: h \geq f, h$ is simple $\}$ ). Then $\int_{X} f d \mu=\sup _{g \in S_{f}} \int_{X} g d \mu=\inf _{h \in V_{f}} \int_{X} h d \mu$, and $f$ is integrable.

Proof. It suffices to prove the part involving $S_{f}$. Let $L=\sup _{x \in X} f(x)$ and, for every fixed $n \in \mathbb{N}$, let $s_{n}(0) \equiv u(0)$, and $s_{n}(t) \equiv u\left(\frac{L i}{2^{n}}\right)$ whenever $\left.t \in] \frac{L(i-1)}{2^{n}}, \frac{L i}{2^{n}}\right]\left(i=1, \ldots, 2^{n}\right)$. We have $\int_{0}^{L} s_{n}(t) d t=\sum_{i=1}^{2^{n}} \frac{L}{2^{n}} u\left(\frac{L}{2^{n}} i\right)$. Put

$$
\begin{aligned}
U_{i}^{(n)} & \equiv\left\{x \in X: f(x)>\frac{L i}{2^{n}}\right\} \\
g_{n} & \equiv \sum_{i=1}^{2^{n}} \frac{L}{2^{n}} \chi_{U_{i}^{(n)}}, \forall n \in \mathbb{N}, i=1,2, \ldots, 2^{n} .
\end{aligned}
$$

Then (Also see [9].) $\int_{X} g_{n} d \mu=\sum_{i=1}^{2^{n}} \frac{L}{2^{n}} \mu\left(U_{i}^{(n)}\right)=\sum_{i=1}^{2^{n}} \frac{L}{2^{n}} u\left(\frac{L}{2^{n}} i\right)$. Taking the supremum, we get

$$
\int_{X} f d \mu=\int_{0}^{L} u(t) d t=\sup _{n} \int_{X} g_{n} d \mu=(o)-\lim _{n} \int_{X} g_{n} d \mu .
$$

If $g \in S_{f}$, then $\int_{X} g d \mu \leq \int_{X} f d \mu$, and so $\int_{X} f d \mu=\sup _{n \in \mathbb{N}} \int_{X} g_{n} d \mu \leq$ $\sup _{g \in S_{f}} \int_{X} g d \mu \leq \int_{X} f d \mu$, which completes the proof.

Proposition 3.13. If $f: X \rightarrow \widetilde{\mathbb{R}}_{0}^{+}$is integrable, then $\int_{X} f d \mu=\sup _{g \in S_{f}} \int_{X} g d \mu$. Conversely, if $f \geq 0$ is such that the quantity $\sup _{g \in S_{f}} \int_{X} g d \mu$ exists in $R$, then $f$ is integrable and $\int_{X} f d \mu=\sup _{g \in S_{f}} \int_{X} g d \mu$.

Proof. The assertion follows by Propositions 3.11 and 3.12.

The following result is easy also.

Proposition 3.14. Let $f: X \rightarrow \widetilde{\mathbb{R}}_{0}^{+}$be an integrable map, $g: X \rightarrow \widetilde{\mathbb{R}}_{0}^{+}$ measurable such that $0 \leq g(x) \leq f(x), \quad \forall x \in X$. Then $g$ is integrable, and $\int_{X} g d \mu \leq \int_{X} f d \mu$

Now we note that if $\mu: X \rightarrow R$ is a mean and $\mathcal{C}_{\infty}(\Omega)$ is as in Theorem 2.4, then there exists a nowhere dense set $\Omega^{\prime} \subset \Omega$ such that $\mu(A)(\omega)$ is real, $\forall \omega \notin \Omega^{\prime}, \forall A \in \mathcal{A}$. 
Proposition 3.15. Let $R \subset \mathcal{C}_{\infty}(\Omega)$ be a Dedekind complete Riesz space where $\Omega^{\prime}$ is as above and set $\mu_{\omega}(A) \equiv \mu(A)(\omega), \forall \omega \notin \Omega^{\prime}$. Assume that $f: X \rightarrow \mathbb{R}$ is an integrable map. Then there exists a meager set $N \subset \Omega$ such that $f$ is integrable with respect to $\mu_{\omega}$ and $\int_{A} f d \mu_{\omega}=\left(\int_{A} f d \mu\right)(\omega), \forall \omega \in N^{c}, \forall A \in$ $\mathcal{A}$.

Proof. Without loss of generality, we can assume that $f$ is nonnegative. First suppose that $f$ is bounded. There exists a sequence of simple functions $\left(s_{n}\right)_{n}$ such that $s_{n} \uparrow f$ and $\int s_{n} d \mu \uparrow \int f d \mu$. So we have, for every $n \in \mathbb{N}$, up to the complement of a meager set, depending only on $X$

$$
\begin{aligned}
0 & \leq\left|\int_{A} f d \mu_{\omega}-\left(\int_{A} f d \mu\right)(\omega)\right| \\
& \leq\left|\int_{A} f d \mu_{\omega}-\int_{A} s_{n} d \mu_{\omega}\right|+\left|\int_{A} s_{n} d \mu_{\omega}-\left(\int_{A} f d \mu\right)(\omega)\right| \\
& =\left|\int_{A} f d \mu_{\omega}-\int_{A} s_{n} d \mu_{\omega}\right|+\left|\left(\int_{A} s_{n} d \mu\right)(\omega)-\left(\int_{A} f d \mu\right)(\omega)\right| \\
& \leq \int_{X} f-s_{n} d \mu_{\omega}+\left(\int_{X} f-s_{n} d \mu\right)(\omega) .
\end{aligned}
$$

Then

$$
\begin{aligned}
0 & \leq\left|\int_{A} f d \mu_{\omega}-\left(\int_{A} f d \mu\right)(\omega)\right| \\
& \leq \limsup _{n} \int_{X} f-s_{n} d \mu_{\omega}+\limsup _{n}\left(\int_{X} f-s_{n} d \mu\right)(\omega) \\
& =\inf _{n} \int_{X} f-s_{n} d \mu_{\omega}+\inf _{n}\left(\int_{X} f-s_{n} d \mu\right)(\omega)=0 .
\end{aligned}
$$

Assume now that $f$ is integrable. By the previous step, there exists a meager set $N^{*}$ such that, $\forall n \in \mathbb{N}, \forall \omega \notin N^{*}, \forall A \in \mathcal{A}$

$$
\int_{A}(f \wedge n) d \mu_{\omega}=\left(\int_{A} f \wedge n d \mu\right)(\omega)
$$

The proof is now analogous to the first part. It will be enough to replace $s_{n}$ with $f \wedge n$.

Now we prove the following theorem. 
Theorem 3.16. Let $f: X \rightarrow \tilde{\mathbb{R}}_{0}^{+}$be an integrable map. Then there exists a meager set $N$ such that for every $A \in \mathcal{A}$ and for every $\omega \notin N$,

$$
\left(\int_{A} f d \mu\right)(\omega) \in(\mu(A) \overline{\operatorname{co}}\{f(x): x \in A\})(\omega) .
$$

Proof. By Proposition 3.15 and classical results we have, up to the complement of a meager set

$$
\begin{aligned}
\left(\int_{A} f d \mu\right)(\omega) & =\int_{A} f d \mu_{\omega} \in \mu_{\omega}(A) \overline{\mathrm{co}}\{f(x), x \in A\} \\
& =\overline{\mathrm{co}}\left\{f(x) \mu_{\omega}(A), x \in A\right\}=(\mu(A) \overline{\mathrm{co}}\{f(x), x \in A\})(\omega) .
\end{aligned}
$$

For the definition of absolute continuity and related remarks, see ([4]).

Proposition 3.17. If $f: X \rightarrow \tilde{\mathbb{R}}_{0}^{+}$is integrable, then the integral $\int . f d \mu$ is absolutely continuous; that is, $(o)-\lim _{n} \int_{A_{n}} f d \mu=0$ whenever $\left(A_{n}\right)_{n}$ is a sequence in $\mathcal{A}$ such that $(o)-\lim _{n} \mu\left(A_{n}\right)=0$.

Proof. The assertion is trivial when $f$ is bounded. So we prove absolute continuity in the general case. Fix $n, k \in \mathbb{N}$, and pick $\left(A_{n}\right)_{n}$, with $(o)-$ $\lim _{n} \mu\left(A_{n}\right)=0$. We have

$$
\begin{aligned}
0 & \leq \int_{A_{n}} f d \mu=\int_{A_{n}}(f \wedge k) d \mu+\int_{A_{n}} f-(f \wedge k) d \mu \\
& \leq \int_{A_{n}}(f \wedge k) d \mu+\int_{X} f-(f \wedge k) d \mu .
\end{aligned}
$$

As $(o)-\lim _{k} \int_{X} f-(f \wedge k) d \mu=0$ and $(o)-\lim _{n} \int_{A_{n}}(f \wedge k) d \mu=0$ for each $k \in \mathbb{N}$, there exist a sequence $\left(r_{k}\right)_{k}$ in $R, r_{k} \downarrow 0$, and a double sequence $\left(r_{n, k}^{\prime}\right)_{n, k}$ in $R, r_{n, k}^{\prime} \downarrow 0(n \rightarrow+\infty, k=1,2, \ldots)$ such that

$$
0 \leq \int_{A_{n}} f d \mu \leq r_{n, k}^{\prime}+r_{k}, \quad \forall n, k \in \mathbb{N} .
$$

It follows that

$$
0 \leq(o)-\limsup _{n \rightarrow+\infty} \int_{A_{n}} f d \mu \leq\left((o)-\limsup _{n \rightarrow+\infty} r_{n, k}^{\prime}\right)+r_{k}=r_{k}, \quad \forall k \in \mathbb{N} .
$$

By the arbitrariness of $k$, we get $(o)-\lim \sup _{n \rightarrow+\infty} \int_{A_{n}} f d \mu=0$ and hence (o) $-\lim _{n \rightarrow+\infty} \int_{A_{n}} f d \mu=0$.

Now we will prove a Vitali-type theorem for our integral. 
Definition 3.18. Let $\left(f_{n}: X \rightarrow \widetilde{\mathbb{R}}\right)_{n}$ be a sequence of integrable functions. We say that $\left(f_{n}\right)_{n}$ is uniformly integrable if $\sup _{n} \int_{X}\left|f_{n}\right| d \mu \in$ Rand (o) $\lim _{n} \sup _{k \geq n}\left(\int_{A_{n}}\left|f_{k}\right| d \mu\right)=0$, whenever $(o)-\lim _{k} \mu\left(A_{k}\right)=0$.

Definition 3.19. Under the same hypotheses and notation as above, we say that $\left(f_{n}\right)_{n}$ converges in $L^{1}$ to $f$ if $(o)-\lim _{n} \int_{X}\left|f_{n}-f\right| d \mu=0$.

Remark 3.20. It is easy to check that $\left(f_{n}\right)_{n}$ converges in $L^{1}$ to $f$ if and only if $\int_{A} f d \mu=(o)-\lim _{n \rightarrow+\infty} \int_{A} f_{n} d \mu$ uniformly with respect to $A \in \mathcal{A}$.

Theorem 3.21. (Vitali's theorem) Under the same notation as above, let $\left(f_{n}\right)_{n}$ be a uniformly integrable sequence of functions, convergent in measure to $f$. Then $f$ is integrable and $\left(f_{n}\right)_{n}$ converges in $L^{1}$ to $f$.

Conversely, every sequence $\left(f_{n}\right)$ of integrable functions, convergent in $L^{1}$ to an integrable map $f$, is convergent in measure to $f$ and uniformly integrable.

Proof. To obtain the integrability of $|f|$, it is enough to prove that

$$
\sup S_{|f|} \equiv \sup \left\{\int_{X} \varphi d \mu: 0 \leq \varphi \leq|f| \text { and } \varphi \text { is simple }\right\} \in R
$$

by virtue of Proposition 3.13. Let $\varphi \in S_{|f|}, \varphi=\sum_{j=1}^{k} c_{j} \chi_{B_{j}}$. Fix $j=$ $1,2, \ldots, k$ and for every $n \in \mathbb{N}$, set $A_{n} \equiv E_{1}^{\left|f-f_{n}\right|}$. If $x \in A_{n}{ }^{c} \cap B_{j}$, we have $\varphi(x)=c_{j} \leq\left|f_{n}(x)\right|+1$ and hence $\int_{B_{j} \cap A_{n}^{c}} \varphi(x) d \mu \leq \int_{B_{j}}\left|f_{n}(x)\right| d \mu+\mu\left(B_{j}\right)$. As to $A_{n} \cap B_{j}$, we have $\int_{B_{j} \cap A_{n}} \varphi(x) d \mu \leq c_{j} \mu\left(A_{n}\right)$. Thus

$$
\begin{gathered}
\int_{B_{j}} \varphi(x) d \mu \leq \int_{B_{j}}\left|f_{n}(x)\right| d \mu+\mu\left(B_{j}\right)+c_{j} \mu\left(A_{n}\right), \\
\int_{X} \varphi(x) d \mu \leq \int_{X}\left|f_{n}(x)\right| d \mu+\mu(X)+\mu\left(A_{n}\right) \sum_{j=1}^{k} c_{j} .
\end{gathered}
$$

By convergence in measure, $(o)-\lim _{n \rightarrow+\infty} \mu\left(A_{n}\right) \sum_{j=1}^{k} c_{j}=0$ and since $n$ is arbitrary, $\int_{X} \varphi d \mu \leq \sup _{n} \int_{X}\left|f_{n}\right| d \mu+\mu(X) \in R$. Since the right hand side does not depend on $\varphi$, (1) follows. So $|f|$ is integrable. By Proposition 3.14, $f^{+}$and $f^{-}$are integrable and so is $f$.

Now fix $\varepsilon>0$ and $n \in \mathbb{N}$. As $f_{n}$ is integrable by hypothesis, so is $f-f_{n}$. 
We have

$$
\begin{aligned}
& \int_{X}\left|f_{n}-f\right| d \mu \leq \int_{\left\{x \in X:\left|f_{n}-f\right| \leq \varepsilon\right\}}\left|f_{n}-f\right| d \mu+\int_{\left\{x \in X:\left|f_{n}-f\right|>\varepsilon\right\}}\left|f_{n}-f\right| d \mu \\
& \quad \leq \int_{X} \varepsilon d \mu+\int_{\left\{x \in X:\left|f_{n}-f\right|>\varepsilon\right\}}\left|f_{n}\right| d \mu+\int_{\left\{x \in X:\left|f_{n}-f\right|>\varepsilon\right\}}|f| d \mu \\
& \quad \leq \varepsilon \cdot \mu(X)+\sup _{k \geq n} \int_{\left\{x \in X:\left|f_{n}-f\right|>\varepsilon\right\}}\left|f_{k}\right| d \mu+\int_{\left\{x \in X:\left|f_{n}-f\right|>\varepsilon\right\}}|f| d \mu .
\end{aligned}
$$

As $(o)-\lim _{n} \mu\left(\left\{x \in X:\left|f-f_{n}\right|>\varepsilon\right\}\right)=0$, by virtue of uniform integrability of $\left(f_{k}\right)_{k}$, integrability of $f$ and absolute continuity of the integral we get

$$
(o)-\lim _{n \rightarrow+\infty}\left[\sup _{k \geq n} \int_{\left\{x \in X:\left|f_{n}-f\right|>\varepsilon\right\}}\left|f_{k}\right| d \mu+\int_{\left\{x \in X:\left|f_{n}-f\right|>\varepsilon\right\}}|f| d \mu\right]=0 .
$$

So there exists a sequence $\left(r_{n}\right)_{n}$ in $R, r_{n} \downarrow 0$ such that

$$
0 \leq \int_{X}\left|f_{n}-f\right| d \mu \leq \varepsilon \cdot \mu(X)+r_{n}, \quad \forall n \in \mathbb{N} .
$$

Thus we obtain

$$
\begin{aligned}
0 & \leq(o)-\limsup _{n \rightarrow+\infty} \int_{X}\left|f_{n}-f\right| d \mu \leq \varepsilon \cdot \mu(X)+(o)-\limsup _{n \rightarrow+\infty} r_{n} \\
& =\varepsilon \cdot \mu(X)+\inf _{n \in \mathbb{N}} r_{n}=\varepsilon \cdot \mu(X) .
\end{aligned}
$$

Since $\varepsilon>0$ was arbitrary, we get $(o)-\lim _{n \rightarrow+\infty} \int_{X}\left|f_{n}-f\right| d \mu=0$.

Conversely, suppose that $\left(f_{n}\right)_{n}$ converges in $L^{1}$ to $f$. Fix $\varepsilon>0$ and set

$$
E_{\varepsilon}^{\left|f-f_{n}\right|} \equiv\left\{x \in X:\left|f_{n}(x)-f(x)\right|>\varepsilon\right\}, \quad \forall n \in \mathbb{N} .
$$

Then

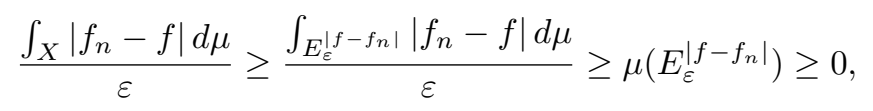

and hence $(o)-\lim _{n} \mu\left(E_{\varepsilon}^{\left|f-f_{n}\right|}\right)=0$.

Now we prove uniform integrability. By convergence in $L^{1}$, it follows immediately that $\sup _{k} \int_{X}\left|f_{k}\right| d \mu \in R$. Let $\left(A_{n}\right)_{n}$ be a sequence in $\mathcal{A}$ such that $(o)-\lim _{n} \mu\left(A_{n}\right)=0$. Fix $n \in \mathbb{N}$. For every $k \geq n$ we have

$$
\int_{A_{n}}\left|f_{k}\right| d \mu \leq \int_{A_{n}}\left|f_{k}-f\right| d \mu+\int_{A_{n}}|f| d \mu \leq \int_{X}\left|f_{k}-f\right| d \mu+\int_{A_{n}}|f| d \mu .
$$


By convergence in $L^{1}$, there exists a sequence $\left(r_{k}\right)_{k}$ in $R, r_{k} \downarrow 0$ such that $\int_{X}\left|f_{k}-f\right| d \mu \leq r_{k} \leq r_{n}$. Thus $\sup _{k \geq n} \int_{A_{n}}\left|f_{k}\right| d \mu \leq r_{n}+\int_{A_{n}}|f| d \mu$. So

$$
0 \leq(o)-\limsup _{n \rightarrow+\infty} \sup _{k \geq n} \int_{A_{n}}\left|f_{k}\right| d \mu \leq \inf _{n} r_{n}+(o)-\limsup _{n \rightarrow+\infty} \int_{A_{n}}|f| d \mu=0
$$

and hence $(o)-\lim _{n \rightarrow+\infty} \sup _{k \geq n} \int_{A_{n}}\left|f_{k}\right| d \mu=0$.

A consequence of Vitali's theorem is the following theorem.

Theorem 3.22. (Lebesgue dominated convergence theorem) Let $\left(f_{n}\right)_{n}, f_{n}$ be a sequence of measurable functions and suppose that there exists an integrable map $h$ such that $\left|f_{n}(x)\right| \leq|h(x)|$ for all $n \in \mathbb{N}$ and almost everywhere with respect to $x$. Furthermore assume that $\left(f_{n}\right)_{n}$ converges in measure to $f$. Then for every $n \in \mathbb{N}, f_{n}$ is integrable and $\left(f_{n}\right)_{n}$ converges in $L^{1}$ to $f$.

Proof. Without loss of generality, we suppose that

$$
\left|f_{n}(x)\right| \leq|h(x)|, \quad \forall n \in \mathbb{N}, \quad \forall x \in X .
$$

By integrability of $|h|$ and Proposition 3.14, $f_{n}$ is integrable for every $n \in \mathbb{N}$. Moreover by virtue of absolute continuity of the integral of $h$, the hypotheses of Theorem 3.21 hold. So the assertion follows.

As a consequence of Theorem 3.22, we prove the following theorem, that is a sufficient condition for the convergence in $L^{1}$, inspired by a well-known result of Scheffé's ([23]):

Theorem 3.23. With the same notation as above, let $\left(f_{n}\right)_{n}: X \rightarrow \widetilde{\mathbb{R}}_{0}^{+}$be a sequence of integrable functions, convergent in measure to a nonnegative integrable mapping $f$. Assume that $\int_{X} f_{n} d \mu(o)$-converges to $\int_{X} f d \mu$. Then $\left(f_{n}\right)_{n}$ converges in $L^{1}$ to $f$.

Proof. Let $h_{n}(x)=f_{n}(x)-f(x), \forall x \in X$. Thus $0 \leq\left[h_{n}(x)\right]^{-} \leq f(x), \forall x$. Let $H_{n}(x)=\left[h_{n}(x)\right]^{-}, \forall x$. Then $f, H_{n}$ are integrable for every $n$ and $\left(H_{n}\right)_{n}$ converges in measure to 0 . By Theorem 3.22, we have $0=(o)-$ $\lim _{n} \int_{X}\left[h_{n}(x)\right]^{-} d \mu$ and so $(o)-\lim _{n} \int_{X}\left[h_{n}(x)\right]^{+} d \mu=(o)-\lim _{n} \int_{X} h_{n} d \mu=0$, by hypothesis. Finally we get

$$
\begin{aligned}
(o)-\lim _{n} \int_{X}\left|h_{n}\right| d \mu= & (o)-\lim _{n} \int_{X}\left[h_{n}(x)\right]^{+} d \mu \\
& +(o)-\lim _{n} \int_{X}\left[h_{n}(x)\right]^{-} d \mu=0 .
\end{aligned}
$$

We now state a version of the monotone convergence theorem. 
Theorem 3.24. With the same notation as above, let $\left(f_{n}\right)_{n}$ be an increasing sequence of non negative integrable maps, convergent in measure to an integrable function $f$. Then $\int_{X} f d \mu=(o)-\lim _{n} \int_{X} f_{n} d \mu$ and therefore $f_{n} \rightarrow f$ in $L^{1}$.

Proof. It is an immediate consequence of Vitali's Theorem.

\section{Countably Additive Case}

If $\mu$ is countably additive, convergence almost everywhere implies convergence in measure; this can be proved along classical lines. Hence we simply state the results. So both Levi's theorem and Fatou's lemma hold.

Proposition 4.1. Let $R$ be a Dedekind complete Riesz space, $\mathcal{A} \subset \mathcal{P}(X)$ a $\sigma$-algebra, and assume that $\mu: \mathcal{A} \rightarrow R$ is a $\sigma$-additive mean. Set

$$
A_{n}^{\varepsilon} \equiv\left\{x \in X:\left|f_{n}(x)-f(x)\right|>\varepsilon\right\}, \quad \forall \varepsilon>0 .
$$

Then, $f_{n}$ converges almost everywhere to $f$ if and only if $\mu\left(\lim \sup _{n} A_{n}^{\varepsilon}\right)=0$, $\forall \varepsilon>0$.

It is easy to prove the following.

Proposition 4.2. Let $R, \mathcal{A}$ and $\mu$ be as above, and assume that $\mu$ is $\sigma$ additive. Then for each sequence $\left(A_{n}\right)$ in $\mathcal{A}$ one has

$$
\mu\left(\liminf _{n} A_{n}\right) \leq \liminf _{n} \mu\left(A_{n}\right) \leq \limsup _{n} \mu\left(A_{n}\right) \leq \mu\left(\limsup _{n} A_{n}\right) .
$$

A straightforward consequence of Proposition 4.2 is the following.

Theorem 4.3. Let $f_{n}, f$ and $\mu$ be as above. If $\left(f_{n}\right)$ converges to $f$ almost everywhere, $\left(f_{n}\right)$ converges to $f$ in measure.

From Theorems 3.24 and 4.3, and by the proceeding as in the classical case, the next theorem follows.

Theorem 4.4. With the same notation and hypotheses as above, let $\left(f_{n}\right)_{n}$ be an increasing sequence of nonnegative measurable maps. Then $f(x) \equiv$ $\lim _{n} f_{n}(x)$ is integrable if and only if $\lim _{n} \int_{X} f_{n} d \mu \in R$ and in this case

$$
\int_{X} f d \mu=(o)-\lim _{n} \int_{X} f_{n} d \mu .
$$

A consequence of Beppo Levi's Theorem is the following version of Fatou's Lemma. 
Theorem 4.5. Let $X, R, \mu$ be as above, $\left(f_{n}\right)_{n}$ a sequence of nonnegative integrable maps, $f(x) \equiv \liminf _{n} f_{n}(x), \forall x \in X$. If $\liminf _{n} \int_{X} f_{n} d \mu \in R$, then $f$ is integrable and $\liminf _{n} \int_{X} f_{n} d \mu \geq \int_{X} f d \mu$.

\section{Radon-Nikodym Theorem}

In this section we give a Greco-type condition for the existence of a RadonNikodym derivative for the monotone integral introduced in the previous section (see [14]). We show that the Radon-Nikodym problem, in general, has no solutions. Indeed, there exist two $\mathbb{R}^{2}$-valued $\sigma$-additive means $\mu$ and $\nu$, with $\nu \ll \mu$, such that there is no function $f: X \equiv\{0,1\} \rightarrow \mathbb{R}$ such that $\nu=\int_{X} f d \mu$.

Let $X \equiv\{0,1\}, \mathcal{A} \equiv \mathcal{P}(X), R \equiv \mathbb{R}^{2}$ (endowed with componentwise ordering), $\mu, \nu: \mathcal{P}(X) \rightarrow \mathbb{R}^{2}$ defined by setting

$$
\mu(\{0\})=(1,0), \quad \mu(\{1\})=(0,1), \quad \nu(\{0\})=(0,1), \quad \nu(\{1\})=(1,0) .
$$

It is easy to check that $\mu$ and $\nu$ are $\sigma$-additive, $\nu$ is absolutely continuous with respect to $\mu$ and $\mu$ is absolutely continuous with respect to $\nu$. However there is no function $f: X \rightarrow \mathbb{R}$ such that $\nu(A)=\int_{A} f d \mu, \forall A \in \mathcal{P}(X)$ for otherwise, we would have $(1,0)=\nu(\{1\})=\int_{\{1\}} f d \mu=f(1) \mu(\{1\})=(0, f(1))$, which is a contradiction.

Furthermore it is easy to see that for every $r>0$ there exists no Hahn decomposition for the map $\nu-r \mu$.

Now we introduce two preliminary lemmas.

Proposition 5.1. Let $\mu, \nu: \mathcal{A} \rightarrow R$ be two means with $\nu \ll \mu$. If there exists an $\mathcal{A}$-measurable function $f: X \rightarrow \widetilde{\mathbb{R}}_{0}^{+}$such that, for every $E \in \mathcal{A}$

$$
\nu(E)=\int_{E} f d \mu
$$

then, for every $r>0$, the set $A_{r}=\{x \in X: f(x)>r\}$ satisfies

(5.1.1) $\nu(E) \geq r \mu(E)$ for every $E \in A_{r} \cap \mathcal{A}$,

(5.1.2) $\nu(E) \leq r \mu(E)$ for every $E \in A_{r}^{c} \cap \mathcal{A}$,

(5.1.3) $(o)-\lim _{r \rightarrow+\infty} \nu\left(A_{r}\right)=0$. 
Proof. $A_{r} \in \mathcal{A}$ for every $r>0$ since $f$ is measurable. Moreover for every $r>0$ and for every $E \in A_{r} \cap \mathcal{A}, F \in A_{r}^{c} \cap \mathcal{A}$ we have

$$
\begin{aligned}
& \nu(E)=\int_{E} f d \mu \geq \int_{E} r d \mu=r \mu(E) \\
& \nu(F)=\int_{F} f d \mu \leq \int_{F} r d \mu=r \mu(F) .
\end{aligned}
$$

This proves (5.1.1) and (5.1.2).

(5.1.3) is a consequence of (5.1.1). In fact (5.1.1) yields

$$
\mu\left(A_{r}\right) \leq \frac{\nu\left(A_{r}\right)}{r} \leq \frac{\nu(X)}{r}, \forall r>0 .
$$

So $(o)-\lim _{r \rightarrow+\infty} \mu\left(A_{r}\right)=0$, and hence $(o)-\lim _{r \rightarrow+\infty} \nu\left(A_{r}\right)=0$.

Proposition 5.2. Let $\mu, \nu: \mathcal{A} \rightarrow R$ be two means with $\nu \ll \mu$. Let $D \equiv\left\{\frac{i}{2^{n}}\right.$, $i, n \in \mathbb{N}\}$. If there exists a decreasing family $\left(A_{r}\right)_{r \in D}$ such that $A_{0}=X$ and satisfying (5.1.1) and (5.1.2), then the function $f: X \rightarrow[0,+\infty]$, defined by $f(x) \equiv \sup \left\{r \in D: x \in A_{r}\right\}$, is integrable and $\nu(E)=\int_{E} f d \mu, \quad \forall E \in \mathcal{A}$.

Proof. $f$ is $\mathcal{A}$-measurable, since, $\forall t>0,\{x \in X: f(x)>t\}=\cup_{r \in D, r>t} A_{r}$. Let $f_{n} \equiv \frac{1}{2^{n}} \sum_{k=1}^{n 2^{n}} \chi_{A_{\frac{k}{2^{n}}}}$, for every $n \in \mathbb{N}$. Then $f \wedge n-f \wedge \frac{1}{2^{n}} \leq f_{n} \leq f, \quad \forall n$. By construction for every $E \in \mathcal{A}$,

$$
\begin{aligned}
\int_{E} f_{n} d \mu & =\frac{1}{2^{n}} \sum_{k=1}^{n 2^{n}} \mu\left(A_{\frac{k}{2^{n}}}\right) \\
& =\sum_{k=1}^{n 2^{n}-1} \frac{k}{2^{n}}\left[\mu\left(A_{\frac{k}{2^{n}}} \cap E\right)-\mu\left(A_{\frac{k+1}{2^{n}}} \cap E\right)\right]+n \mu\left(A_{n} \cap E\right) \\
& \leq \sum_{k=1}^{n 2^{n}-1}\left[\nu\left(A_{\frac{k}{2^{n}}} \cap E\right)-\nu\left(A_{\frac{k+1}{2^{n}}} \cap E\right)\right]+n \nu\left(A_{n} \cap E\right) \leq \nu(E) .
\end{aligned}
$$

So $\sup _{n} \int_{X} f_{n} d \mu \leq \nu(X) \in R$ and thus

$$
\sup _{n} \int_{X}(f \wedge n) d \mu \leq \sup _{n} \int_{X}\left(f_{n}+1\right) d \mu \leq \nu(X)+\mu(X) .
$$

So by Proposition 3.11, $f$ is integrable and hence, by Proposition $3.8, f \cdot \chi_{E}$ 
is integrable, $\forall E \in \mathcal{A}$. Thus

$$
\begin{aligned}
(o)-\lim _{n}\left[\int_{E}(f \wedge n) d \mu-\int_{E}\left(f \wedge \frac{1}{2^{n}}\right) d \mu\right] & =(o)-\lim _{n} \int_{E}(f \wedge n) d \mu \\
& =\int_{E} f d \mu,
\end{aligned}
$$

and therefore $(o)-\lim _{n} \int_{E} f_{n} d \mu=\int_{E} f d \mu$ and $\int_{E} f d \mu \leq \nu(E), \quad \forall E \in \mathcal{A}$. On the other hand,

$$
\begin{aligned}
\int_{E} f_{n} d \mu= & \sum_{k=1}^{n 2^{n}-1} \frac{k+1}{2^{n}}\left[\mu\left(A_{\frac{k}{2^{n}}} \cap E\right)-\mu\left(A_{\frac{k+1}{2^{n}}} \cap E\right)\right]+n \mu\left(A_{n} \cap E\right)+ \\
& -\frac{1}{2^{n}} \sum_{k=1}^{n 2^{n}-1}\left[\mu\left(A_{\frac{k}{2^{n}}} \cap E\right)-\mu\left(A_{\frac{k+1}{2^{n}}} \cap E\right)\right] \\
\geq & \nu\left(A_{\frac{1}{2^{n}}} \cap E\right)-\nu\left(A_{n} \cap E\right)-\frac{1}{2^{n}}\left(\mu\left(A_{\frac{k}{2^{n}}}\right)-\mu\left(A_{n} \cap E\right)\right) .
\end{aligned}
$$

Taking the (o)-limits as $n \rightarrow \infty$, we obtain $\int_{E} f d \mu=\nu(E)$.

A consequence of Proposition 5.1 and 5.2 is the following Radon-Nikodym Theorem.

Theorem 5.3. Let $\mu, \nu: \mathcal{A} \rightarrow R$ be two means with $\nu \ll \mu$. Then the following are equivalent:

(5.3.a) there exists an $\mathcal{A}$-measurable function $f: X \rightarrow \widetilde{\mathbb{R}}_{0}^{+}$such that, for every $E \in \mathcal{A}$ we have $\nu(E)=\int_{E} f d \mu$,

(5.3.b) there exists a family $\left(A_{r}\right)_{r>0}$ of measurable sets such that for every $r>0$

(5.3.b.1) $\nu(E) \geq r \mu(E)$ for every $E \in A_{r} \cap \mathcal{A}$,

(5.3.b.2) $\nu(E) \leq r \mu(E)$ for every $E \in A_{r}^{c} \cap \mathcal{A}$.

The following is a different formulation of Theorem 5.3.

Theorem 5.4. Let $\mu, \nu: \mathcal{A} \rightarrow R$ be two means with $\nu \ll \mu$. Then the following are equivalent:

(5.4.a) there exists a $\mathcal{A}$-measurable function $f: X \rightarrow \widetilde{\mathbb{R}}_{0}^{+}$such that, for every $E \in \mathcal{A}$ we have $\nu(E)=\int_{E} f d \mu$, 
(5.4.b) for every $r>0$ the measure $\nu-r \mu$ admits a Hahn decomposition, namely there exist two disjoint measurable sets $\left(B_{r}, C_{r}\right)$ such that, $\forall E \in$ $\mathcal{A}$

$$
\begin{aligned}
& (\nu-r \mu)^{+}(E)=(\nu-r \mu)\left(E \cap B_{r}\right) \\
& (\nu-r \mu)^{-}(E)=(\nu-r \mu)\left(E \cap C_{r}\right) .
\end{aligned}
$$

Proof. (5.4.a) $\Longrightarrow$ (5.4.b)

By Theorem 5.3, there exists a family $\left(A_{r}\right)_{r>0}$ of measurable sets such that, for every $r>0$

(5.3.b.1) $\nu(E) \geq r \mu(E)$ for every $E \in A_{r} \cap \mathcal{A}$,

(5.3.b.2) $\nu(E) \leq r \mu(E)$ for every $E \in A_{r}^{c} \cap \mathcal{A}$.

Set $B_{r} \equiv A_{r}, C_{r} \equiv A_{r}^{c}$. For every $E \in A_{r} \cap \mathcal{A}$ we have

$$
\begin{aligned}
(\nu-r \mu)^{+}(E) & =(\nu-r \mu)^{+}\left(E \cap A_{r}\right)+(\nu-r \mu)^{+}\left(E \cap A_{r}^{c}\right) \\
& =(\nu-r \mu)^{+}\left(E \cap A_{r}\right)=(\nu-r \mu)\left(E \cap A_{r}\right)
\end{aligned}
$$

from (5.3.b.1), since $(\nu-r \mu)(F) \leq 0, \forall F \in E \cap A_{r}^{c} \cap \mathcal{A}$. So we obtain, for every $E \in \mathcal{A},(\nu-r \mu)^{+}(E)=(\nu-r \mu)\left(E \cap B_{r}\right)$. Analogously for each $E \in \mathcal{A}$, $(\nu-r \mu)^{-}(E)=(\nu-r \mu)\left(E \cap C_{r}\right)$. (5.4.b) $\Longrightarrow(5.4 . \mathrm{a})$

It is easy to check that, if (5.4.b) holds, then (5.3.b.1.) and (5.3.b.2.) are satisfied. The assertion follows by Proposition 5.2.

\section{References}

[1] C. D. Aliprantis and O. Burkinshaw, Locally solid Riesz spaces, Academic Press, 1978.

[2] S. J. Bernau, Unique representation of Archimedean lattice groups and normal Archimedean lattice rings, Proc. London Math. Soc., 15 (1965), 599-631.

[3] A. Boccuto, Riesz spaces, integration and sandwich theorems, Tatra Mountains Math. Publ., 3 (1993), 213-230.

[4] A. Boccuto, Abstract integration in Riesz spaces, Tatra Mountains Math. Publ., 5 (1995), 107-124.

[5] J. K. Brooks and A. Martellotti, On the De Giorgi-Letta integral in infinite dimensions, Atti Sem. Mat. Fis. Univ. Modena, 4 (1992), 285-302. 
[6] G. Choquet, Theory of capacities, Annales de l'Istitut de Fourier, 5 (195354), 131-295.

[7] M. Congost Iglesias, Medidas y probabilidades en estructures ordenadas, Stochastica, 5 (1981), 45-68.

[8] R. Cristescu, On integration in ordered vector spaces and on some linear operators, Rend. Circ. Mat. Palermo, Suppl., 33 (1993), 289-299.

[9] E. De Giorgi and G. Letta, Une notion générale de convergence faible des fonctions croissantes d'ensemble, Ann. Scuola Sup. Pisa, 33 (1977), 61-99.

[10] D. Denneberg, Non-additive measure and integral, Kluwer Acad. Publ., 1994.

[11] M. Duchoň and B. Riečan, On the Kurzweil-Stieltjes integral in ordered spaces, to appear on Tatra Mountains Math. Publ.

[12] I. Gilboa and D. Schmeidler, Additive representation of non-additive measures and the Choquet integral, Ann. Oper. Res., 52 (1994), 43-65.

[13] G. H. Greco, Integrale monotono, Rend. Sem. Mat., Univ. Padova, 57 (1977), 149-166.

[14] G. H. Greco, Un teorema di Radon-Nikodym per funzioni d'insieme subadditive, Ann. Univ. Ferrara, 27 (1981), 13-19.

[15] J. Haluška, On integration in complete vector lattices, Tatra Mountains Math. Publ., 3 (1993), 201-212.

[16] M. C. Isidori, A. Martellotti and A. R. Sambucini, The monotone integral, preprint, 1995.

[17] W. A. J. Luxemburg and A. C. Zaanen, Riesz Spaces I, North-Holland Publishing Co., 1971.

[18] P. Maličký, Random variables with values in a vector lattice (mean value and conditional mean value operators), Acta Math. Univ. Comenianae, 52-53 (1987), 249-263.

[19] P. Mcgill, Integration in vector lattices, J. Lond. Math. Soc., 11 (1975), 347-360.

[20] E. J. Mcshane, A Riemann-type integral that includes Lebesgue-Stieltjes, Bochner and stochastic integrals, Mem. Amer. Math. Soc., 88 (1969). 
[21] B. Riečan, On the Kurzweil integral for functions with values in ordered spaces I, Acta Math. Univ. Comenian., 56-57 (1989), 411-424.

[22] B. Riečan and M. Vrábelová, On the Kurzweil integral for functions with values in ordered spaces II, Math. Slovaca, 43 (1993), 471-475.

[23] H. Scheffe, A useful convergence theorem for probability distributions, Ann. Math. Stat., 18 (1947) 434-438.

[24] J. Šipoš, Integral with respect to a pre-measure, Math. Slov., 29 (1979), $141-155$

[25] B. Z. Vulikh, Introduction to the theory of partially ordered spaces, Wolters-Noordhoff Sci. Publ., Groningen, 1967.

[26] J. D. M. Wright, Stone-algebra-valued measures and integrals, Proc. Lond. Math. Soc., 19 (1969), 107-122.

[27] J. D. M. Wright, Measures with values in a partially ordered vector space, Proc. London Math. Soc., 25 (1972), 675-688.

[28] A. C. Zaanen, Riesz spaces II, North-Holland Publishing Co., 1983. 